https://helda.helsinki.fi

Formation of $\mathrm{HArF}$ in solid Ar revisited : Are mobile vacancies involved in the matrix-site conversion at $30 \mathrm{~K}$ ?

Khriachtchev, Leonid

American Institute of Physics

2004

Journal of chemical physics. - New York, NY :. - ISSN 0021-9606. 120 (2004) : 7, s. 3353-3357.

http://link.aip.org/link/?JCP/120/3353/1

Downloaded from Helda, University of Helsinki institutional repository.

This is an electronic reprint of the original article.

This reprint may differ from the original in pagination and typographic detail.

Please cite the original version. 


\title{
Formation of HArF in solid Ar revisited: Are mobile vacancies involved in the matrix-site conversion at $30 \mathrm{~K}$ ?
}

\author{
Leonid Khriachtchev, ${ }^{\text {a) }}$ Antti Lignell, and Markku Räsänen \\ Laboratory of Physical Chemistry, P.O. Box 55, FIN-00014, University of Helsinki, Finland
}

(Received 19 November 2003; accepted 21 November 2003)

\begin{abstract}
The HArF molecule can occupy in solid Ar thermally unstable and stable configurations, and their microscopic structure is not understood at the moment. We present additional experimental results on the formation of two HArF configurations and analyze them with emphasis on possible reactions of the unstable configuration with matrix vacancies to form the stable configuration. We conclude that the existing computational scenarios do not describe fully the present experimental data. In order to explain qualitatively the experimental results, two tentative models are discussed. The first model is based on local mobility of matrix vacancies produced during photolysis and the second model considers isomerization of the HArF @ $\mathrm{Ar}_{n}$ supermolecule. More importantly, the present results constitute the experimental basis for future theoretical studies. (c) 2004 American Institute of Physics. [DOI: 10.1063/1.1641016]
\end{abstract}

\section{INTRODUCTION}

A few years ago, our group reported the first neutral ground-state chemical compound with argon, HArF. ${ }^{1}$ This compound was prepared using vacuum-UV photolysis of $\mathrm{HF}$ in solid $\mathrm{Ar}$ at $7 \mathrm{~K}$ and annealing at $\sim 20 \mathrm{~K}$ promoting the $\mathrm{H}+\mathrm{Ar}+\mathrm{F} \rightarrow \mathrm{HArF}$ reaction and leading to the characteristic $\mathrm{H}-\mathrm{Ar}$ stretching absorption bands at 1965.7, 1969.4, and $1972.3 \mathrm{~cm}^{-1}$. This triplet absorption was found to decrease and disappear upon annealing above $28 \mathrm{~K}$, which was tentatively connected with chemical reactions of HArF with thermally mobile fragments like fluorine atoms. Later, ${ }^{2}$ we found that HArF exhibits additional blueshifted $\mathrm{H}-\mathrm{Ar}$ stretching absorption bands at 2016.3 and $2020.8 \mathrm{~cm}^{-1}$ which correspond to a species with larger thermal stability limited only by degrading of the matrix structure above $40 \mathrm{~K}$. This doublet absorption was assigned to HArF in a thermally relaxed solid-state configuration (matrix site), and we call it "stable" $\mathrm{HArF}$ in order to distinguish it from the "unstable" HArF absorbing at $\sim 1970 \mathrm{~cm}^{-1}$. It was experimentally shown that the annealing-induced conversion of unstable HArF to stable HArF was very probable, and this relaxation was the basis of our experimental model. ${ }^{2}$ It was also found that complexation with normal matrix impurities (including residual HF) did not cause the observed conversion. ${ }^{2}$ Interestingly, similar modification of the matrix-site structure has been recently found for HArF complexed with $\mathrm{N}_{2}$ in solid argon. ${ }^{3}$ The studies of complexed HArF show large complexationinduced shifts of the $\mathrm{H}-\mathrm{Ar}$ stretching absorption supporting the idea about high sensitivity of this strongly ionic and weakly bound species to local organization of the surrounding. ${ }^{3,4}$ This property can be employed to study fine details of solid-state hosts.

The observed thermal modification of the HArF absorption bands is a remarkable solid-state phenomenon, and it needs theoretical interpretation. A number of simulations ap-

${ }^{a)}$ Electronic mail: leonid.khriachtchev@helsinki.fi peared quickly after the experimental report. ${ }^{5-7}$ Bihary et al. assigned the stable absorptions to HArF in a compact (single-substitutional, SS) matrix site and the unstable absorptions to HArF in a loose (double-substitutional, DS) site. ${ }^{5}$ Their assignment was based on comparison between the experimental and theoretical absorption frequencies. In contrast, Jolkkonen et al. have assigned the stable absorption bands to HArF in a loose site and the unstable absorptions to HArF in a compact site. ${ }^{6}$ The blueshift of the stable HArF bands was connected with specific complexation of one matrix Ar atom with the HArF molecule from the $\mathrm{H}$ side, which was energetically more favorable in the loose configuration. Qualitatively similar conclusions were derived in the Nemukhin group. ${ }^{7}$ It is seen that the theoretical models connect the difference between the stable and unstable configurations of HArF with their substitutional numbers. This means that the thermal reorganization of the system involves a mobile vacancy: Loosing of the vacancy in the model by Bihary et $a l^{5}$ and accepting a vacancy in the model by Jolkkonen et $a .^{6}$

In this work, we report additional experiments on the formation of two HArF configurations in solid Ar and analyze the results with emphasis on reorganization processes involving matrix vacancies. For the present work, we had several motivations. First, we wanted to evaluate the existing theoretical models using new experimental data, and hence we constructed the experiments to be sensitive to the theoretical predictions. Second, this new experimental data could provide a basis for future theoretical studies of this phenomenon, which is definitely needed. Because none of the existing computational models fully describes the experimental evidence, we qualitatively discuss possible ways to explain the present results.

\section{EXPERIMENTAL RESULTS}

The HF/Ar solid mixtures were studied in a closed-cycle helium cryostat (APD, DE 202A) at temperatures down to 7 


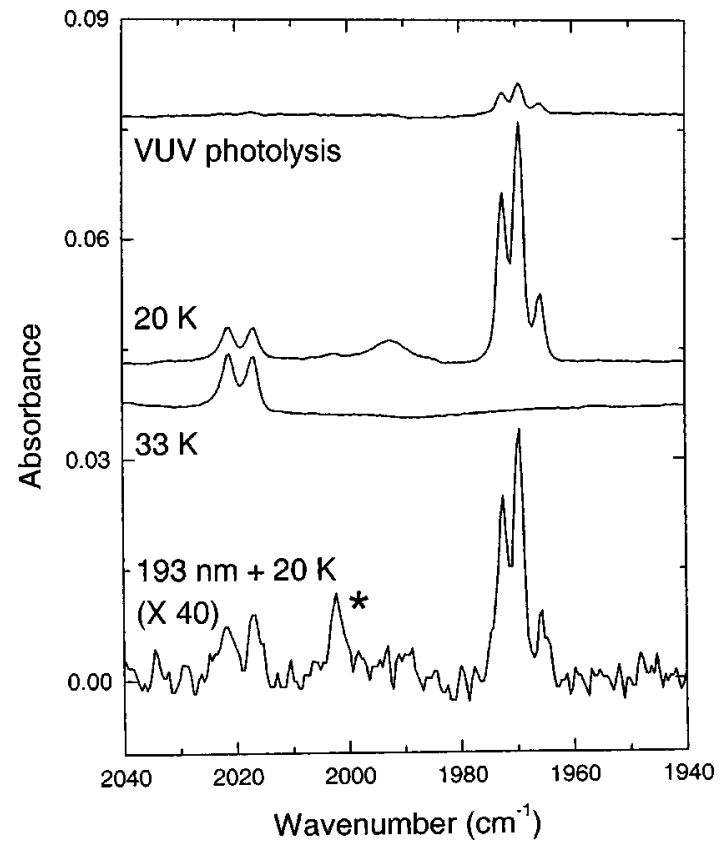

FIG. 1. Formation of HArF in solid Ar. Shown are (from top to bottom) the IR absorption spectrum of the $\mathrm{H}-\mathrm{Ar}$ stretching vibration after VUV photolysis of the HF/Ar matrix, the spectrum after annealing at $20 \mathrm{~K}$, the spectrum after annealing of the same sample at $33 \mathrm{~K}$, the spectrum after $193 \mathrm{~nm}$ irradiation of the same sample and annealing at $20 \mathrm{~K}$. Note a multiplication factor of 40. The spectra were measured at $7 \mathrm{~K}$. The band at $2004 \mathrm{~cm}^{-1}$ (marked with an asterisk) is assigned to the HArF $\cdots \mathrm{N}_{2}$ complex (Ref. 3).

K. The samples were deposited onto a cold CsI substrate by passing Ar gas (99.9999\%, AGA) over an HF-pyridine polymer (Fluka) at room temperature. Photolysis of HF was performed with a $\mathrm{Kr}$ continuum lamp (Opthos) emitting in the $127-160 \mathrm{~nm}$ spectral interval. The IR absorption spectra in the 4000-400 $\mathrm{cm}^{-1}$ region were recorded with a Nicolet 60 SX FTIR spectrometer using a resolution of $1 \mathrm{~cm}^{-1}$. Our $\mathrm{HF} / \mathrm{Ar}$ matrixes were quite monomeric, and the doublet band of HF monomer at 3962.5 and $3953.8 \mathrm{~cm}^{-1}$ dominated in the IR absorption spectra in agreement with the literature data. ${ }^{8}$ $193 \mathrm{~nm}$ radiation of an excimer laser (MSX-250, MPB) was used to decompose synthesized HArF molecules.

Figure 1 presents various steps of the HArF formation in solid Ar. Some amount of HArF is seen already after vacuum-ultraviolet (VUV) photolysis indicating the "direct" $\mathrm{H}+\mathrm{Ar}+\mathrm{F} \rightarrow \mathrm{HArF}$ reactions as observed earlier for other rare-gas hydrides ( $\mathrm{HXeNCO}$ and $\mathrm{HKrCl}){ }^{9,10}$ In particular, this formation of HArF during photolysis indicates shortrange light-induced travel of the dissociating fragments as discussed elsewhere. ${ }^{11}$ It should be noticed that the photolysis favors unstable configuration absorbing at $\sim 1970 \mathrm{~cm}^{-1}$ rather than the stable one. Annealing at $20 \mathrm{~K}$ further increases concentration of unstable HArF and leads to the appearance of stable HArF. In addition, a broad feature around $1985 \mathrm{~cm}^{-1}$, which has escaped from discussions so far, ${ }^{1-3}$ also belongs most probably to HArF in solid Ar. Annealing at $33 \mathrm{~K}$ bleaches the unstable HArF absorption bands completely and increases the stable HArF concentration. According to the experimental model suggested in Ref. 2, this behavior is a result of the rather synchronous conversion of unstable HArF to stable HArF and reactions of HArF mol- ecules with thermally mobilized fragments. The latter contribution is not fundamentally important but it can explain the relatively low absorbance of stable HArF as compared with unstable HArF; and this decay channel has been supported by experiments on other rare-gas hydrides $(\mathrm{HXeH}$ and $\mathrm{HKrCl}){ }^{12,13}$ The relatively low absorption of the stable configuration can be also connected with a change of the $\mathrm{H}-\mathrm{Ar}$ stretching intensity of HArF upon specific interactions with Ar atoms. ${ }^{6,14}$ Stable HArF produced upon annealing at $33 \mathrm{~K}$ was decomposed by $193 \mathrm{~nm}$ radiation, and the irradiated sample was annealed at $20 \mathrm{~K}$ (see the lower trace in Fig. 1). Important for the present study, the proportion between the unstable and stable HArF concentrations is very similar to that obtained after the first annealing at $20 \mathrm{~K}$.

It was found earlier that the increase of deposition temperature from 7 to $18 \mathrm{~K}$ shifts the disappearance of the unstable HArF configuration to somewhat higher temperatures, approximately from 28 to $31 \mathrm{~K}^{2}$. This effect is demonstrated in Figs. 2(a) and 2(b). It is seen that the partial stabilization of unstable HArF is accompanied with the delay of the increase of the stable HArF concentration. It was evidently interesting to try deposition above the conversion temperature, however, we found that deposition above $30 \mathrm{~K}$ did not lead to isolation of HF monomers. This is why we chose another procedure, namely, deposition of the matrix at $18 \mathrm{~K}$ and postdeposition (prephotolysis) annealing at $33 \mathrm{~K}$. Such sample was photolyzed by the $\mathrm{Kr}$ lamp at $7 \mathrm{~K}$ and then annealed at $20 \mathrm{~K}$, and the upper line in Fig. 3 presents the resulting spectrum. The lower line shows a reference spectrum obtained in a "normal" manner (deposition at 7 $\mathrm{K}$-VUV photolysis - annealing at $20 \mathrm{~K}$ ). It is seen that the two spectra are remarkably similar. Moreover, at $32 \mathrm{~K}$ the unstable HArF configuration decomposes independently of the prephotolysis annealing at $33 \mathrm{~K}$. Thus, prephotolysis annealing at $33 \mathrm{~K}$ did not change processes connected with the HArF modifications as compared with the situation without the annealing. Figure 2(c) presents the correlation between the integrated absorption bands of unstable HArF and stable $\mathrm{HArF}$ for five experiments with varying deposition condition. For each experiment, the increase of the stable HArF concentration correlates in some temperature region with the decrease of the unstable HArF concentration, hence supporting the conversion mechanism. The proportion between maximal concentrations of the unstable and stable HArF molecules is similar in various experiments indicating that secondary reactions with thermally mobilized fragments are probably not of major importance here.

\section{DISCUSSION}

The model allowing HArF in single- and doublesubstitutional matrix sites is based on the following arguments. HF in solid Ar may occupy two different matrix sites, which are possibly interstitial and single-substitutional. ${ }^{8}$ After photolysis, the $\mathrm{H}$ and $\mathrm{F}$ atoms can occupy either interstitial or single-substitutional positions. Upon thermal mobilization of these atoms, the following reactions lead to HArF in different local arrangements:

$$
\mathrm{H}^{\mathrm{Int}}+\mathrm{Ar}^{\mathrm{SS}}+\mathrm{F}^{\mathrm{SS}} \rightarrow \mathrm{HArF}^{\mathrm{DS}},
$$



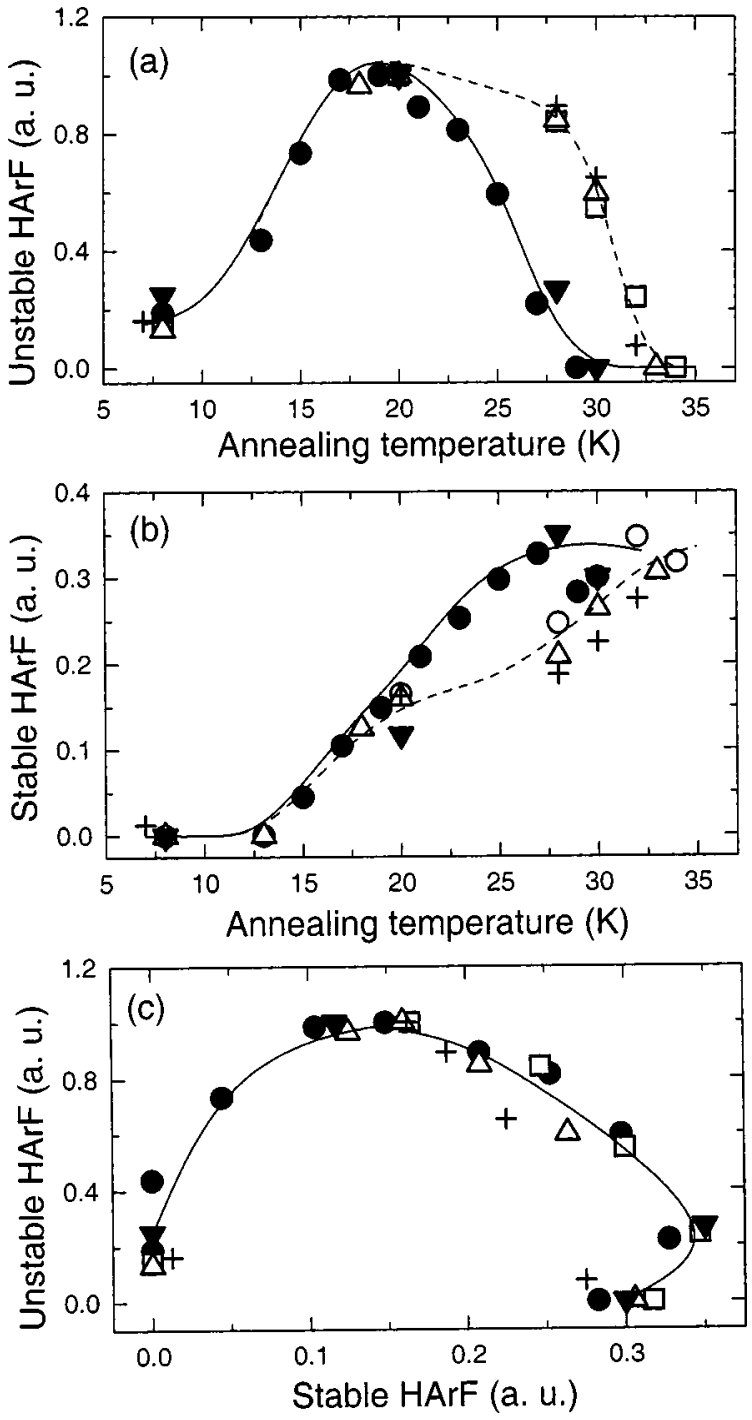

FIG. 2. Integrated IR absorption intensities of (a) unstable and (b) stable configurations of HArF in solid Ar as a function of the annealing temperature. The samples were annealed at a given temperature during several minutes and then cooled down to $7 \mathrm{~K}$ to measure the spectrum. The data points of five experiments are shown with different symbols. The closed symbols (guided by solid lines) correspond to deposition at $7 \mathrm{~K}$, the open symbols (guided by dotted lines) represent the data for deposition at $18-20 \mathrm{~K}$ and crosses give the data points for the prephotolysis annealing at $33 \mathrm{~K}$. (c) Correlation between integrated IR absorption intensities of unstable and stable HArF in solid Ar obtained in various experiments.

$$
\begin{aligned}
& \mathrm{H}^{\mathrm{Int}}+\mathrm{Ar}^{\mathrm{SS}}+\mathrm{F}^{\mathrm{Int}} \rightarrow \mathrm{HArF}^{\mathrm{SS}}, \\
& \mathrm{H}^{\mathrm{SS}}+\mathrm{Ar}^{\mathrm{SS}}+\mathrm{F}^{\mathrm{Int}} \rightarrow \mathrm{HArF}^{\mathrm{DS}},
\end{aligned}
$$

where the superscripts Int, SS, and DS denote the interstitial, single-substitutional, and double-substitutional configurations, respectively. It is assumed here that the interstitial atoms are mobile only. It follows that HArF can, in principle, occupy single- and double-substitutional matrix sites. Under this assumption, the conversion of unstable HArF to stable HArF would mean, depending on the assignment, either the reaction of the unstable form with a diffusing vacancy or its loosing of the attached vacancy. However, these two events are not supported by the present experiments. Indeed, the reaction with a vacancy at $30 \mathrm{~K}$ may have no barrier. Nev-

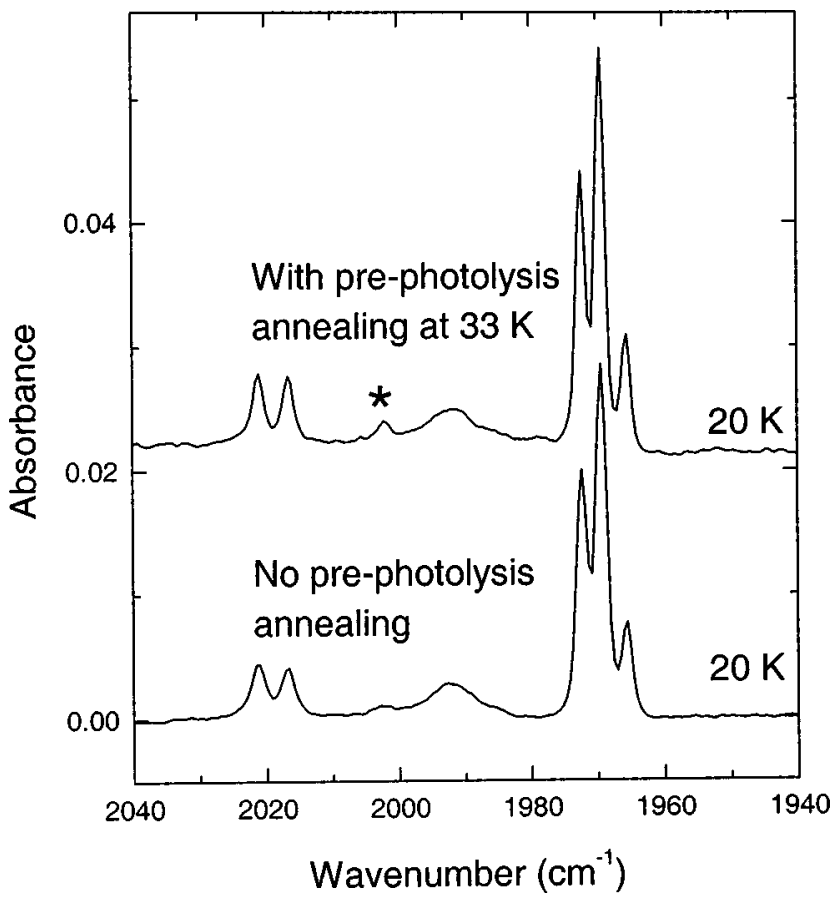

FIG. 3. IR absorption spectra of the H-Ar stretching vibration of HArF in solid Ar for deposition at $18 \mathrm{~K}$ and prephotolysis annealing at $33 \mathrm{~K}$ (the upper trace) and for deposition at $7 \mathrm{~K}$ (no prephotolysis annealing, the lower trace). The spectra were obtained by VUV photolysis of HF/Ar matrixes and annealing at $20 \mathrm{~K}$. The spectra were measured at $7 \mathrm{~K}$. Note the remarkable similarity of these two spectra. The band at $2004 \mathrm{~cm}^{-1}$ (marked with an asterisk) is assigned to the HArF $\cdots \mathrm{N}_{2}$ complex (Ref. 3).

ertheless, there is no evidence for diffusion of matrix vacancies at this temperature. In our experiments (see Figs. 1 and 3 ), annealing at $33 \mathrm{~K}$ (i.e., above the conversion temperature) did not change the proportion between the stable and unstable configurations appearing upon additional annealing at $20 \mathrm{~K}$ and, moreover, it did not prevent disappearance of the unstable configuration at $32 \mathrm{~K}$. This means that the matrix morphology did not change at $33 \mathrm{~K}$ with respect to formation and conversion of HArF. If the matrix vacancies were globally mobile at $30 \mathrm{~K}$ as suggested in Ref. 6 they would be trapped in the main part after the $33 \mathrm{~K}$ annealing. In other words, after preliminary annealing at $33 \mathrm{~K}$ the matrix vacancies could not start moving at $30 \mathrm{~K}$ to promote the reaction. On the other hand, loosing of the vacancy needed for the assignment by Bihary et al. ${ }^{5}$ does not contradict with the result shown in Fig. 3 because the loose (unstable) configuration can be formed via reactions (1) and (3) independently of the vacancy concentration. However, it is unclear how $\mathrm{HArF}^{\mathrm{SS}}$ photodecomposed at $193 \mathrm{~nm}$ can yield so efficiently $\mathrm{HArF}^{\mathrm{DS}}$ upon annealing at $20 \mathrm{~K}$ (see Fig. 1). In addition, the vacancy escape from the loose configuration to the matrix should possess some energy barrier, and this barrier is intuitively similar (if not higher) to the barrier of a vacancy jump inside the matrix. This barrier should be a matter of theoretical evaluation.

These conclusions based on our experiments agree with the literature data on vacancy mobility in Ar crystals. ${ }^{15}$ The activation energy for vacancy diffusion in solid Ar is 160 $170 \mathrm{meV}(1810-1980 \mathrm{~K})$. The experimentally observable mobility of vacancies at $30 \mathrm{~K}$ leads to the unrealistic Arrhen- 
ius preexponential factor $\left(\geqslant e^{60} \mathrm{~s}^{-1}\right)$. It is difficult to expect that the preexponential factor for vacancy activation in solid $\mathrm{Ar}$ is much larger than the corresponding value for hydrogen atoms in rare-gas solids $\left(\sim e^{23}-e^{25} \mathrm{~s}^{-1}\right.$, which corresponds to the typical phonon frequency). ${ }^{13,16}$ To remind ourselves, hydrogen mobility in $\mathrm{Kr}$ and $\mathrm{Xe}$ solids are characterized by activation energies of $\sim 66$ and $120 \mathrm{meV}$, and the experimentally observable mobility starts at $\sim 28$ and $38 \mathrm{~K}$, respectively. ${ }^{13,16,17}$ Jolkkonen et al. calculated the barrier for a vacancy jump in a perfect Ar lattice to be $8 \mathrm{~kJ} / \mathrm{mol}(\sim 82$ $\mathrm{meV}){ }^{6}$ which is two times smaller that the experimental value $(160-170 \mathrm{meV})^{15}$ demonstrating that the theoretical procedure does not accurately describe experiment. This consideration confirms that formation of unstable HArF via reaction (2) does not meet the experimental data because there is no clear mechanism to transfer it to the loose (stable) configuration. This might indicate that formation of HArF in a tight site is protected by a relatively high barrier due to sufficient reorganization of the local matrix surrounding required for the process. As another possibility, both sites of $\mathrm{HF}$ in an Ar matrix are single-substitutional.

We can propose tentative models agreeing with the experimental data, i.e., without relying on vacancy diffusion. First, we start from HF localized in a single-substitutional matrix site and assume that photodissociation of HF leads to interstitial $\mathrm{H}$ and $\mathrm{F}$ atoms and the vacancy finds a vicinal position. The closely separated $\mathrm{H}$ and $\mathrm{F}$ atoms can form $\mathrm{HArF}^{\mathrm{SS}}$, which is an unstable configuration like in Ref. 6. This assumption is consistent with the minor production of the stable (DS) configuration during photolysis (see Fig. 1). Upon annealing at $20 \mathrm{~K}$, the close $\mathrm{H}+\mathrm{F}$ pair reacts with an $\mathrm{Ar}$ atom to produce additional unstable HArF. In this image, the triplet band structure of the unstable configuration might originate from the specific (discrete) position of the vicinal vacancy with respect to the HArF molecule. As compared with global motion, local (short-range) mobility has different (less strict) energetic restriction. ${ }^{18} \mathrm{HArF}^{\mathrm{SS}}$ prepared from $\mathrm{HF}^{\mathrm{SS}}$ is connected with the vacancy by strain, and the vacancy can move to $\mathrm{HArF}$ and react with it at rather low temperatures (below 30-32 K) hence producing the stable configuration. This process of strain-assisted lowtemperature mobility was discussed in the literature with respect to geminate recombination in matrix-isolation studies. ${ }^{19}$ Some production of the stable form at $20 \mathrm{~K}$ is explained by distribution of the activation energies for the local vacancy mobility. The dependencies in Fig. 2 suggest a change of the activation energy of the conversion process upon different deposition conditions, which is also reasonable.

The second scheme agreeing with the experimental observations is the following. We assume that the loose HArF configuration has two forms, with and without formation of the $\mathrm{Ar} \cdots \mathrm{HArF}$ complex, appearing as the stable and unstable forms. These two forms can be separated by low energy barrier, and the transition from the higher-energy form to the lower-energy form can occur at $\sim 30 \mathrm{~K}$. Considering HArF in Ar surrounding ( $\mathrm{HArF} @ \mathrm{Ar}_{n}$ ) as a supermolecule, we discuss here two of its isomers. In fact, two stable configurations of HArF in loose matrix site (DS and T-shaped ac- cording to their nomenclature) were obtained computationally. ${ }^{6}$ In this image, HF precursor in a SS matrix site photodissociates into the $\mathrm{H}+\mathrm{F}$ pair, one of the atoms occupying the SS site (probably F) and the second atom being interstitial. The preferable formation of the higherenergy configuration upon photolysis and low-temperature annealing should be commented. This observation may indicate that the formation of the higher-energy configuration involves smaller reorganization of the surrounding and hence has a smaller formation barrier. The extensive reorganization of the local matrix morphology is energetically demanding meaning a higher dynamical barrier. A similar effect was observed in the $\mathrm{H}+\mathrm{SO}_{2}$ reaction in solid rare gases where annealing-induced formation of the higher-energy $\mathrm{HSO}_{2}$ isomer dominated over formation of the low-energy HOSO isomer. $^{20}$

\section{CONCLUDING REMARKS}

In this work, we presented additional experimental data on formation of various HArF configurations in solid Ar. It seems that these experimental results are not fully understood within the existing theoretical models. In order to agree with the experimental evidence, we proposed two tentative models. The first model involves local mobility of vacancies produced during photolysis and the second one assumes thermal isomerization of the HArF @ $\mathrm{Ar}_{n}$ supermolecule. Our present experiments are unable to distinguish which of these two models is preferable. Moreover, additional qualitative images can be probably introduced. The present results contribute to the experimental basis of future theoretical studies.

A number of additional experimental observations remained unanswered. For example, it is unclear if the formation of HArF involves local or global mobility of atoms. This interesting question has been particularly discussed by Bihary et al. in their very recent article. ${ }^{21}$ The barriers for various reactions discussed in our work are a challenge for theory. The broad spectral feature at $\sim 1985 \mathrm{~cm}^{-1}$ belongs most probably to some configuration of HArF in solid $\mathrm{Ar}$, and its formation should be considered as well as the fine splitting of the doublet and triplet bands. With certainty, there is room for further experimental and theoretical efforts.

\section{ACKNOWLEDGMENTS}

The Academy of Finland supported this work. A.L. is a member of the graduate school Laskemo (Ministry of Education, Finland). Elena Savchenko is thanked for information of vacancy mobility. We also thank Alexander Nemukhin, Zsolt Bihary, R. Benny Gerber, and Mika Pettersson for additional information and discussions on their theoretical studies.

\footnotetext{
${ }^{1}$ L. Khriachtchev, M. Pettersson, N. Runeberg, J. Lundell, and M. Räsänen, Nature (London) 406, 874 (2000).

${ }^{2}$ L. Khriachtchev, M. Pettersson, A. Lignell, and M. Räsänen, J. Am. Chem. Soc. 123, 8610 (2001).

${ }^{3}$ A. Lignell, L. Khriachtchev, M. Pettersson, and M. Räsänen, J. Chem. Phys. 118, 11120 (2003).

${ }^{4}$ S. A. C. McDowell, J. Chem. Phys. 118, 4066 (2003).
} 
${ }^{5}$ Z. Bihary, G. M. Chaban, and R. B. Gerber, J. Chem. Phys. 116, 5521 (2002).

${ }^{6}$ S. Jolkkonen, M. Pettersson, and J. Lundell, J. Chem. Phys. 119, 7356 (2003).

${ }^{7}$ A. V. Nemukhin et al. (unpublished).

${ }^{8}$ D. T. Anderson and J. S. Winn, Chem. Phys. 189, 171 (1994).

${ }^{9}$ M. Pettersson, L. Khriachtchev, J. Lundell, S. Jolkkonen, and M. Räsänen, J. Phys. Chem. A 104, 3579 (2000)

${ }^{10}$ L. Khriachtchev, M. Pettersson, J. Lundell, and M. Räsänen, J. Chem. Phys. 114, 7727 (2001).

${ }^{11}$ J. Lundell, L. Khriachtchev, M. Pettersson, and M. Räsänen, Low Temp. Phys. 26, 680 (2000).

${ }^{12}$ L. Khriachtchev, M. Pettersson, H. Tanskanen, and M. Räsänen, Chem. Phys. Lett. 359, 135 (2002).

${ }^{13}$ L. Khriachtchev, M. Saarelainen, M. Pettersson, and M. Räsänen, J. Chem. Phys. 118, 6403 (2003).
${ }^{14}$ N. Runeberg, M. Pettersson, L. Khriachtchev, J. Lundell, and M. Räsänen, J. Chem. Phys. 114, 836 (2001).

${ }^{15}$ R. O. Simmons, Mater. Chem. Phys. 50, 124 (1997), and references therein.

${ }^{16}$ L. Khriachtchev, H. Tanskanen, M. Pettersson, M. Räsänen, V. Feldman, F. Sukhov, A. Orlov, and A. F. Shestakov, J. Chem. Phys. 116, 5708 (2002).

${ }^{17}$ J. Eberlein and M. Creuzburg, J. Chem. Phys. 106, 2188 (1997).

${ }^{18}$ L. Khriachtchev, M. Pettersson, S. Pehkonen, E. Isoniemi, and M. Räsänen, J. Chem. Phys. 111, 1650 (1999).

${ }^{19}$ V. A. Apkarian and N. Schwentner, Chem. Rev. 99, 1481 (1999).

${ }^{20}$ E. Isoniemi, L. Khriachtchev, J. Lundell, and M. Räsänen, Phys. Chem. Chem. Phys. 4, 1549 (2002).

${ }^{21}$ Z. Bihary, G. M. Chaban, and R. B. Gerber, J. Chem. Phys. 119, 11278 (2003). 